University of South Carolina

Scholar Commons

Faculty Publications

Physics and Astronomy, Department of

$5-8-2000$

\title{
Low Temperature Anomaly in Mesoscopic Kondo Wires
}

P. Mohanty

Richard A. Webb

University of South Carolina - Columbia, webbra@mailbox.sc.edu

Follow this and additional works at: https://scholarcommons.sc.edu/phys_facpub

Part of the Physics Commons

\section{Publication Info}

Published in Physical Review Letters, Volume 84, Issue 19, 2000, pages 4481-4484.

Mohanty, P. and Webb, R.A. (2000). Low Temperature Anomaly in Mesoscopic Kondo Wires. Physical Review Letters, 84(19), 4481-4484. doi: 10.1103/PhysRevLett.84.4481

(C) 2000 The American Physical Society.

This Article is brought to you by the Physics and Astronomy, Department of at Scholar Commons. It has been accepted for inclusion in Faculty Publications by an authorized administrator of Scholar Commons. For more information, please contact digres@mailbox.sc.edu. 


\title{
Low Temperature Anomaly in Mesoscopic Kondo Wires
}

\author{
P. Mohanty ${ }^{1}$ and R. A. Webb ${ }^{2}$ \\ ${ }^{1}$ Condensed Matter Physics 114-36, California Institute of Technology, Pasadena, California 91125 \\ ${ }^{2}$ Center for Superconductivity Research, University of Maryland, College Park, Maryland 20742
}

(Received 27 April 1998; revised manuscript received 19 January 2000)

\begin{abstract}
We report the observation of an anomalous magnetoresistance in extremely dilute quasi-onedimensional AuFe wires at low temperatures, along with a hysteretic background at low fields. The Kondo resistivity does not show the unitarity limit down to the lowest temperature, implying uncompensated spin states. We suggest that the anomalous magnetoresistance may be understood as the interference correction from the accumulation of geometric phase in the conduction electron wave function around the localized impurity spin.
\end{abstract}

PACS numbers: 75.20.Hr, 73.23.-b, 03.65.Bz

A localized magnetic moment interacts with the conduction electrons in a metal resulting in a logarithmic increase of the resistivity as the temperature is lowered. This is known as the Kondo effect [1]. Below the Kondo temperature, $T_{K}$, an electron cloud begins to screen the impurity until its spin is completely compensated, forming a singlet state at low temperature. The nature of this state and the extent of the screening cloud has been studied for decades. Recently this effect has been explored in mesoscopic systems in an attempt to understand whether the screening is affected by the finite sample size [2-4], including high temperature large-concentration experiments on layered Kondo systems [5], and 2D films [3,6]. Interference effects in mesoscopic Kondo systems containing impurity concentrations $c>50 \mathrm{ppm}$ do not generally contribute significantly to the measured magnetoresistance or resistivity because of the strong suppression of long range phase coherence due to spin-flip scattering. In spite of its relevance to mesoscopic systems, a complete study of the low temperature magnetoresistance in very dilute alloys $(c<10 \mathrm{ppm})$, where the Kondo screening length is comparable to the phase coherence length $L_{\phi}$, has not been done. In this regime, an interference experiment which can reveal new information on the development of the Kondo screening cloud is possible. The three-dimensional character of the local dipolar magnetic field from the impurity spin coupled with an externally applied field should provide an additional interference contribution to the electron wave function. This is analogous to the Berry phase effect predicted for coherent electrons in a ring [7] traversing in an externally applied 3D magnetic field texture concentric with the ring.

In this paper, we report the magnetoresistance and the temperature dependence of the resistivity down to $38 \mathrm{mK}$ for five quasi-1D AuFe wires in the concentration range of $3<c<10 \mathrm{ppm}$. We determine both the spin-flip scattering rate and the phase decoherence rate by fitting the low field magnetoresistance to standard weak localization (WL) theory [8]. We find that the unitarity limit corresponding to the formation of the singlet state is not yet reached at our lowest temperature [9] in spite of the fact that AuFe Kondo systems are known to have a Kondo temperature of $1 \mathrm{~K}[10,11]$. At intermediate fields we observe a negative magnetoresistance that is characteristic in temperature dependence and shape of an interference correction, and different from the expected standard Kondo magnetoresistance. At low temperatures this magnetoresistance shows hysteresis which vanishes if the magnetic field is swept to a larger value or if the temperature is increased. We argue that our data is not consistent with a spin glass model but rather with a new interference correction similar to a Berry phase effect [7].

Our studies are done on pure $(99.9995 \%)$ samples of gold $(\mathrm{Au})$ before, and after, the ion implantation of 3-10 ppm of iron (Fe) impurities. This provides a clear advantage over earlier works on layered or flashevaporated samples in that the contribution to the magnetoresistance at various field scales coming solely from the magnetic impurities could be easily identified. Sample dimensions, diffusion constant $D$, and $L_{\phi}$ measured after implantation are given in Table I. These samples are quasi-1D, since $w, t \ll L_{T}, L_{\phi}$, where $L_{T}=\sqrt{\hbar D / k_{B} T}$ is the thermal diffusion length. The Kondo contribution to the resistivity $\Delta \rho(T)$ is found to have the expected logarithmic increase [1]: $\Delta \rho(T)=A-B \ln (T)$ (see Fig. 1), after the subtraction of the electron-electron interaction contribution [4] measured before the ion implantation, which has the expected theoretical value [8], $\Delta \rho_{e e} \simeq\left(2 e^{2} R^{2} w t / h L^{2}\right) L_{T}$.

The total scattering rate $1 / \tau$ relevant for resistance is $1 / \tau=1 / \tau_{n}+1 / \tau_{s} ; 1 / \tau_{n}$ is the nonmagnetic scattering rate. The phase-breaking rate $1 / \tau_{\phi}$ in the presence of magnetic scattering is given by $\tau_{\phi}^{-1}=2 \tau_{s}^{-1}+\tau_{\phi \text { (nonmag) }}^{-1}$. Figure 2 displays the temperature dependence of the magnetic scattering rate $1 / \tau_{s}$ obtained from WL measurements [5,6] for samples AuFe1 and AuFe2. 1/ $\tau_{s}$ is obtained from WL after subtracting the inelastic rate $1 / \tau_{\phi \text { (nonmag) }}$ due to nonmagnetic sources, measured in the same Au wires before ion implantation. The $1 / \tau_{\phi}$ correction term does not produce the observed behavior seen in Fig. 2 because $1 / \tau_{s}$ 
TABLE I. Sample parameters shown in Figs. 1-5.

\begin{tabular}{lccccccrc}
\hline \hline Sample & $\begin{array}{c}w \\
(\mathrm{~nm})\end{array}$ & $\begin{array}{c}t \\
(\mathrm{~nm})\end{array}$ & $\begin{array}{c}L \\
(\mu \mathrm{m})\end{array}$ & $\begin{array}{c}R \\
(\Omega)\end{array}$ & $\begin{array}{c}D \\
\mathrm{~m}^{2} / \mathrm{s}\end{array}$ & $\begin{array}{c}L_{\phi} \\
(\mu \mathrm{m})\end{array}$ & $\begin{array}{c}c \\
(\mathrm{ppm})\end{array}$ & $B$ \\
\hline AuFe1 & 180 & 40 & 155 & 393 & 0.02 & 1.9 & 10.9 & 0.52 \\
AuFe2 & 120 & 40 & 155 & 599 & 0.02 & 2.2 & 7.1 & 0.29 \\
AuFe3 & 100 & 35 & 155 & 803 & 0.02 & 1.7 & 6.0 & 0.24 \\
AuFe4 & 210 & 135 & 4120 & 783 & 0.07 & 5.0 & 3.3 & 0.16 \\
AuFe5 & 120 & 135 & 2750 & 1300 & 0.05 & 3.0 & 10.1 & 0.46 \\
\hline \hline
\end{tabular}

is much larger than $1 / \tau_{\phi}$ in the corresponding clean system. The maxima near $0.2-0.4 \mathrm{~K}$ represent the previously observed resonant spin-flip scattering processes $[6,12]$.

As shown in Fig. 1, the unitarity limit is not reached down to $40 \mathrm{mK}$, even in the presence of disorder and a finite magnetic field required to quench WL, both of which should help form the singlet state. This is consistent with earlier observations $[4,11]$. The impurity spin is thus not completely screened. However, at a larger magnetic field, a resistivity plateau is observed corresponding perhaps to the unitarity limit [see Fig. 3(a)]. The plateau shifted to higher temperatures with increasing magnetic field. Additionally, we observed a maximum around $T_{K}$ [see Fig. 3(b)]. This observation is consistent with earlier experiments on $(\mathrm{LaCe}) \mathrm{Al}_{2}$ and $(\mathrm{LaCe}) \mathrm{B}_{6}$ [13], consequently explained by a wave description of the spin density [14]. This implies that there is a substantial spin polarization around the impurities with a potential $V(r)=V_{0} \cos \left(2 k_{F} r\right) / r^{3}$. The local magnetic field of the spin polarization can be on the order of a Tesla within a couple of nanometers from the impurity, though it is negligible on the scale of the typical interimpurity distance of $\sim 10 \mathrm{~nm}$. The strength of this potential $V_{0}$ is experimentally known to be very large for $\mathrm{AuFe}$, decreasing exponentially with increasing concentration $c$ [15]. Thus, there are strong local magnetic fields for purer samples with longer $L_{\phi}$. NMR measurements of the conduction-electron spin density around $\mathrm{Fe}$ atoms in a $\mathrm{Cu}$ matrix also find a nonvanishing radial component above and below $T_{K}$ [16].

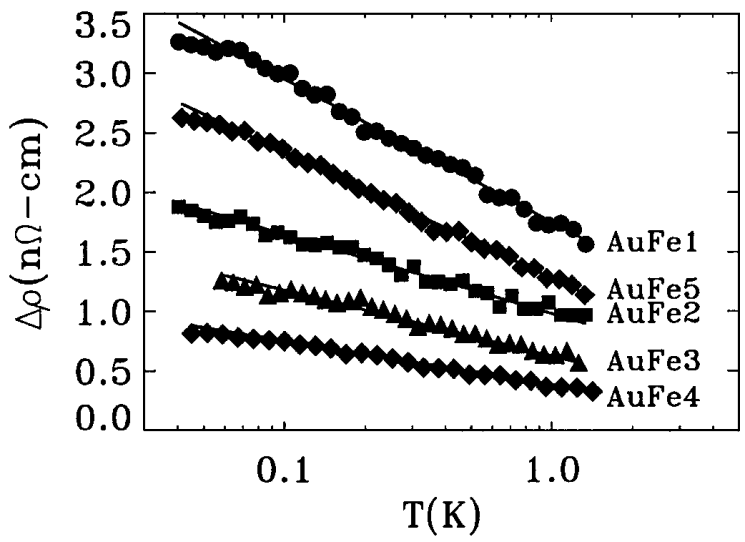

FIG. 1. $\Delta \rho(T)$ for samples AuFe1-AuFe5 at a finite field. The solid lines are fits to $\ln T$.
That there exists a distribution of local magnetic fields from the impurity spins is further confirmed by the observation of hysteresis in the low field MR. As shown in Fig. 4, the background of the WL curve is asymmetric with a positive or negative slope depending on the field history. Hysteresis disappears at high temperatures, typically between 0.4 and $1.5 \mathrm{~K}$, depending on the sample. In contrast to what is observed in a spin glass, we find this hysteresis to be stronger for systems with longer $L_{\phi}$ (hence for lower concentration samples). Hysteresis is expected for a spin glass system below $T_{g}$; so if it were a spin glass, we would have observed stronger hysteresis for higher concentration samples, contrary to our data. Our experiment suggests that hysteresis arises because of different realizations of the three-dimensional local field distribution. As the sample gets cold, impurity spins freeze out in random orientations, providing a particular configuration for the local field distribution. This distribution is modified by a magnetic field due to spin alignment. Magnetic field cycling between \pm 1 T removes the hysteresis and flattens the background of the low field MR, while cycling between $\pm 0.05 \mathrm{~T}$ does not.

All of our samples are in the single-impurity regime and the logarithmic increase of resistivity scales with concentration. It is unlikely that these systems behave like a spin glass for a number of reasons: (a) In AuFe, spin glass behavior is not observed for $c \ll 100 \mathrm{ppm}$, as is well known [17]; (b) second, spin glass temperature $T_{g}$ for a system with 3-10 ppm Fe in Au would be $1 \mathrm{mK}$ or lower; (c) the

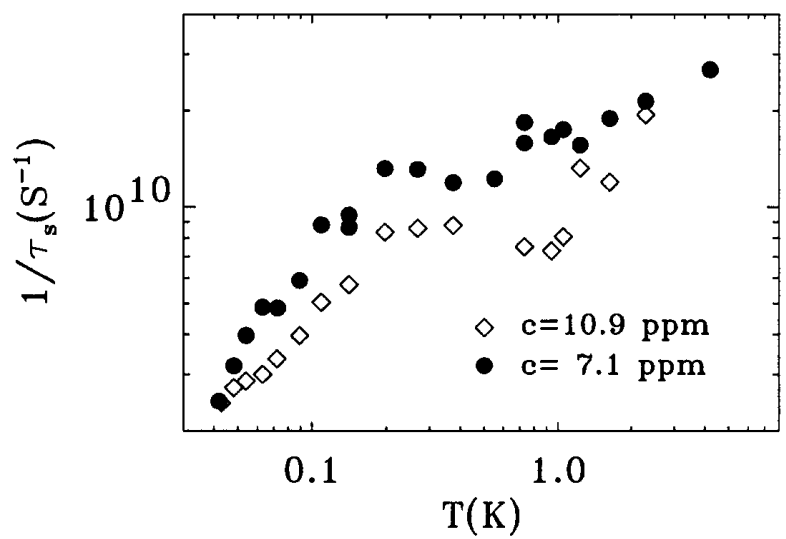

FIG. 2. Spin scattering rate $1 / \tau_{s}$ for samples AuFe1 (diamonds) and AuFe2 (solid circles). 


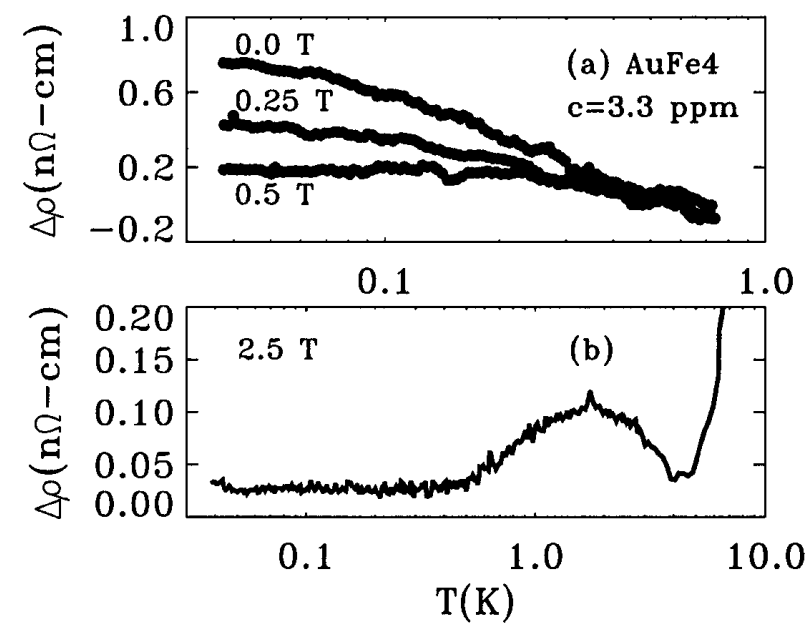

FIG. 3. (a) Resistivity saturation, and (b) the maximum in the Kondo resistivity at $2.5 \mathrm{~T}$.

resistivity maximum expected for a spin glass is also not seen in Fig. 1; (d) the possibility of inhomogeneous pockets of impurities, or clustering, is ruled out by measuring different segments in a sample. The observed behavior is found to be independent of the choice of the segment, suggesting a homogeneous mechanism. For these reasons, the spin glass formation can be ruled out.

High field magnetoresistance of a representative sample, $\mathrm{AuFe}$, is shown in Fig. 5(a). WL is observed at a field scale of $B<0.03 \mathrm{~T}$. At high fields, due to the cyclotron orbits of the electrons, a classical MR is expected: $\Delta R_{c} / R \sim\left(\omega \tau_{e}\right)^{2}$, with $\omega \sim e B / m$, and $\tau_{e}$ being the electron mean free path. This classical $B^{2}$ dependence is displayed in Fig. 5(b), which is subtracted out in Fig. 5(a). At the intermediate field scale $(\sim 1 \mathrm{~T})$, we observe a negative magnetoresistance in all of our samples at $T<T_{K}$ that is very sensitive to temperature. Theoretically, in the standard Kondo model, one expects a negative MR due to the suppression of the spin-flip scattering by the alignment of the spins with the field: $\Delta R_{2} / R \sim(g S \beta)^{2}(H / T)^{2}$, where $\beta$ is the Bohr magneton. The data is not described by this

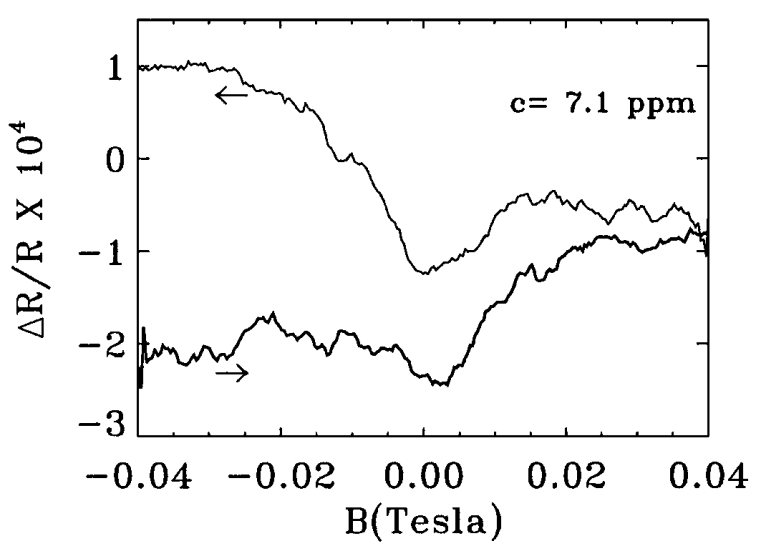

FIG. 4. Typical hysteresis observed in AuFe2 at $140 \mathrm{mK}$. Field sweep directions are marked. contribution, as evident in the shape of the MR at various temperatures. We have observed this anomalous MR in all of our samples along with the WL dip at zero field. At $40 \mathrm{mK}$, the conductance change, $\Delta G=\Delta R / R^{2}$, for all of our samples in units of $e^{2} / h$ is $\sim 0.001, \sim 0.002,0.018$, 0.028, and 0.004 for samples AuFe1 through AuFe5, respectively.

Earlier experiments on higher concentration AuFe samples [3,4] revealed a behavior compatible with the standard expected form, and different from what we observe. Above $T_{K}$, the standard high field magnetoresistance is essentially a function of the thermal average of the local moment in the field direction $\left\langle S_{Z}\right\rangle$. As the temperature is increased, the field scale increases with the height of the MR decreasing, ultimately becoming flat at a very high temperature due to thermal fluctuations of the localized spin. This behavior is observed in 2D Kondo films of AuFe at 1.4 and $4.2 \mathrm{~K}$ [3]. However, in another experiment on AuFe wires [4] with a much higher concentration of Fe impurities $(\sim 50 \mathrm{ppm})$, temperature dependence of the MR was not studied. There are two important characteristics of our low temperature MR, different from the bulk Kondo behavior. First, the magnetoresistance as a function of temperature cannot be explained by $\left\langle S_{Z}\right\rangle$, since the field scale is expected to grow with increasing temperature while conserving area under the curve. Second, $\left\langle S_{Z}\right\rangle$ as a function of temperature is
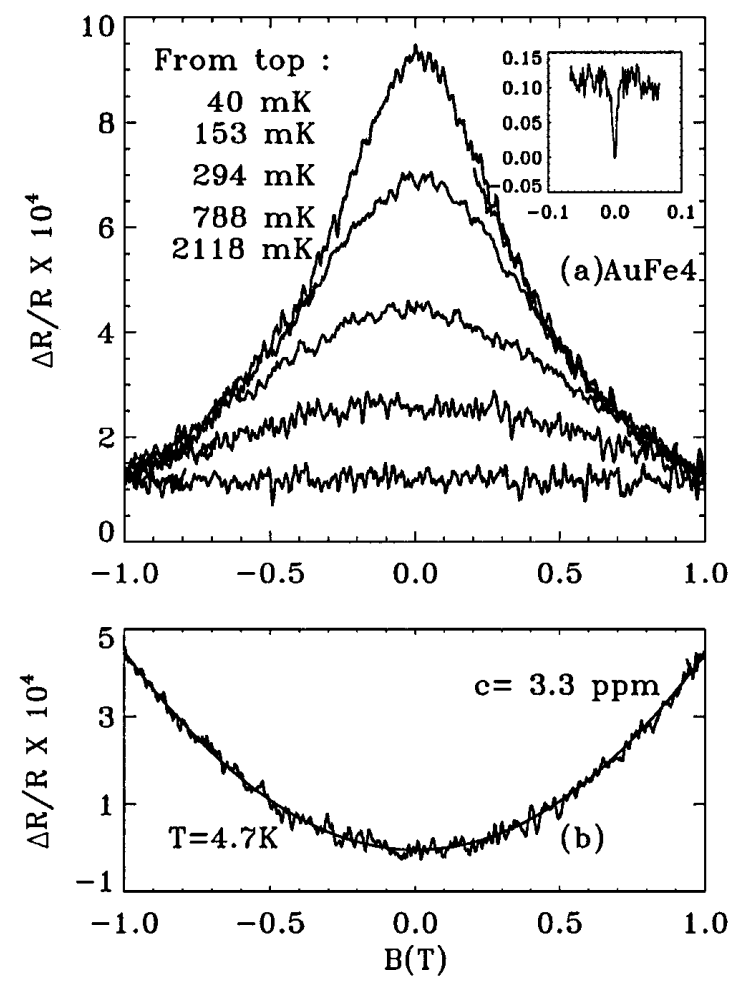

FIG. 5. (a) Magnetoresistance of a representative sample, AuFe4, after the subtraction of the classical magnetoresistance [shown in (b)]. The inset of (a) shows the weak antilocalization contribution at $520 \mathrm{mK}$. 
expected to increase with decreasing temperature, becoming flat at low temperatures, whereas the dependence shown in Fig. 5(a) displays no saturation down to $40 \mathrm{mK}$. High concentrations of impurities in the earlier experiment on AuFe wires [4] and high temperature range in the experiments on 2D AuFe films [3] imply a very short $\tau_{\phi}$ in these systems, yielding nonmesoscopic bulk behavior. The local magnetic field due to polarization in these high concentration samples is expected to be extremely weak, in contrast to our samples

It is clear from our resistivity and scattering rate measurements that the long range polarization of the conduction electrons around the localized spin is effective at low temperatures for our low-concentration mesoscopic systems. From our observation of hysteresis, we believe that this polarization or the local magnetic field causes the anomalous high field magnetoresistance. Furthermore, the shape of the magnetoresistance and its temperature dependence are very much similar to what is expected from a quasi-1D interference effect [8], which suggests a similarity to weak localization. These effects were seen in long $L_{\phi}$ samples, implying an essential role played by the phase coherence of electrons. Considering all this, we propose a connection of this new interference correction to Berry phase.

It is possible for the phase coherent mesoscopic Kondo wires to show a weak-localizationlike magnetoresistance driven by a geometric phase $\Gamma=\int_{t_{i}}^{t_{f}} \mathbf{A}_{g} \cdot d \mathbf{R}$, similar to the standard weak localization driven by the AharonovBohm phase [7]. $\mathbf{A}_{g}$ is the geometric gauge potential, and $\mathbf{R}$ is the position vector describing the tip of the spin. The spin part of the wave function of the phase coherent electron picks up a geometric phase as it aligns along the local magnetic field of the uncompensated spin. This is further helped by disorder in the sample [7], since the electron spends more time around the spin than it would in a ballistic sample. The corresponding geometric phase is equal to half of the solid angle subtended by the area enclosed by the tip of the electron spin vector due to its evolution in a closed loop. A complementary path, going in the opposite direction, will contribute an opposite phase shift. Interference of two such paths around the local field results in a correction to conductivity, analogous to the anticipated Berry phase correction in a ring structure. There are no oscillations as in the Aharonov-Bohm effect, but just half a period in resistivity, because the maximum Berry phase acquired is $\pi$, half of the maximum solid angle of $2 \pi$. An externally applied perpendicular field aligns the electron spin. If the spin is completely aligned along the external field, the solid angle subtended by the tip of the spin is zero, resulting in the complete suppression of the Berry phase correction.

Berry phase changes sign under time reversal. This leads to a contribution similar to the Cooperon propagator in
WL. Correction to the resistance contains the disorder average of all possible loops acquiring Berry phase. As temperature is increased, $L_{\phi}$ (which includes spin fluctuations) reduces greatly, thus reducing the magnetoresistance correction as seen in Fig. 5(a). This dependence is similar to that of WL. In the spirit of WL, a geometric length $L_{B}$ can be introduced, which is the length scale over which the net accumulated geometric phase is on the order of $\pi . L_{B}$ may be defined by $\Delta R_{g} / R^{2} \sim\left(e^{2} / \hbar\right) L_{B} / L$. For the data from the sample AuFe4 [shown in Fig. $5(\mathrm{a})$ ] at $40 \mathrm{mK}$, the geometric length $L_{B} \sim 18 \mu \mathrm{m}\left(L_{\phi} \sim 3 \mu \mathrm{m}\right.$ at $\left.40 \mathrm{mK}\right)$, implying that within $L_{\phi}$ the acquired (disorder-averaged) geometric phase is on the order of $\pi L_{\phi} / L_{B} \sim \pi / 6$ for this sample.

In summary, we have observed an unusual temperature dependence of the magnetoresistance along with hysteresis in quasi-one-dimensional disordered Kondo systems at $T<T_{K}$. We believe that this arises from the adiabatic evolution of the phase coherent electron around the impurity spin, which results in a Berry phase effect.

We thank B. Altshuler, H. Fukuyama, D. Loss, P. Schwab, J. Schwarz, and A. Zawadowski for conversations. This work is supported by the NSF (DMR9510416) and the ARO (DAAG559710330).

[1] A. C. Hewson, The Kondo Problem to Heavy Fermions (Cambridge University Press, Cambridge, England, 1993).

[2] G. Chen and N. Giordano, Phys. Rev. Lett. 66, 209 (1991); M. A. Blachly and N. Giordano, Phys. Rev. B 46, 2951 (1992); 51, 12537 (1995).

[3] N. Giordano, Phys. Rev. B 53, 2487 (1996).

[4] V. Chandrasekhar et al., Phys. Rev. Lett. 72, 2053 (1994).

[5] R. P. Peters et al., Phys. Rev. Lett. 60, 1093 (1988).

[6] C. Van Haesendonck, J. Vranken, and Y. Bruynseraede, Phys. Rev. Lett. 58, 1968 (1987).

[7] D. Loss, P. Goldbart, and A. V. Balatsky, Phys. Rev. Lett. 65, 1655 (1990); D. Loss, H. Schoeller, and P. Goldbart, Phys. Rev. B 48, 15218 (1993); 59, 13328 (1999).

[8] B. L. Altshuler and A. G. Aronov, in Electron-Electron Interaction in Disordered Systems, edited by A. L. Efros and M. Pollak (Elsevier, Amsterdam, 1985).

[9] B. L. Altshuler (private communication).

[10] R. P. Peters et al., Phys. Rev. Lett. 58, 1964 (1987).

[11] M. D. Daybell, in Magnetism V, edited by H. Suhl (Academic Press, New York, 1973).

[12] G. Frossati et al., Physica (Amsterdam) 84B, 33 (1976).

[13] W. Felsch and K. Winzer, Solid State Commun. 13, 569 (1973); Z. Phys. B 25, 269 (1976).

[14] M. Weber, Solid State Commun. 22, 627 (1977).

[15] J. C. Liu et al., Solid State Commun. 17, 595 (1975).

[16] J. B. Boyce et al., Phys. Rev. Lett. 32, 61 (1974).

[17] P. J. Ford et al., Phys. Rev. B 2, 1547 (1970). 